\title{
Efecto combinado de los cambios de salinidad y la exposición al cadmio en las respuestas fisiológicas de Isognomon alatus (Bivalvia: Isognomonidae)
}

\author{
Juan Manuel Polo-Osorioi, ${ }^{1, *}$, Néstor Hernando Campos² \\ ${ }^{1}$ Maestría en Ciencias-Biología, Línea de Biología Marina, Universidad Nacional de Colombia, sede Caribe, Santa Marta, Colombia \\ ${ }^{2}$ CECIMAR, Universidad Nacional de Colombia, sede Caribe, Santa Marta, Colombia
}

\begin{abstract}
Resumen
Se evaluó el efecto combinado de los cambios de salinidad y la exposición al cadmio en las respuestas fisiológicas de Isognomon alatus, especie con potencial biomonitor por su abundancia y resistencia a los cambios de salinidad, temperatura y $\mathrm{pH}$. Se recolectaron 200 individuos que se separaron en el laboratorio en tres grupos y se aclimataron durante un mes en condiciones de salinidad de 36, 27 y 18 UPS, respectivamente. Posteriormente, los individuos se trasladaron en grupos de cinco según el nivel de salinidad a tres tanques con condiciones salinas de 36, 27 y 18 UPS; en cada experimento se aplicaron dos choques salinos y un control de salinidad. El experimento para determinar el efecto del cadmio se realizó una vez con agua libre de metales y otra con una concentración no letal de $1 \mu \mathrm{g} / \mathrm{L}$ de cloruro de cadmio. Se evaluaron las diferencias entre las respuestas fisiológicas con base en las tasas de filtración, respiración y de excreción de amonio, comparando los controles y los tratamientos. Los resultados evidenciaron diferencias tanto en los efectos individuales de los choques salinos como frente a la exposición al cadmio; asimismo, se observó un efecto sinérgico importante, que permitió concluir que la respuesta de la especie como biomonitor depende de las condiciones de salinidad del ecosistema y sus dinámicas. (C) 2016. Acad. Colomb. Cienc. Ex. Fis. Nat.

Palabras clave: Biomonitora; Cadmio; Isognomon alatus; Respuestas fisiológicas.

Mixed effect of salinity and cadmium exposure on physiological responses in Isognomon alatus (Bivalvia: Isognomonidae)

Abstract

We evaluated the mixed effect of changes in salinity and cadmium exposure on the physiological responses of Isognomon alatus, considered as potential biomonitoring species for its abundance and resistance to salinity changes, temperature and $\mathrm{pH}$. Two hundred individuals were taken to the laboratory and acclimated for one month at salinities of 36, 27 and 18 UPS. In each experiment we used two salt shocks and a salinity control. To determine the effect of cadmium the experiment was performed once with free water and then with metals at a non-lethal concentration of $1 \mu \mathrm{g} / \mathrm{L}$ of cadmium chloride. We evaluated the differences in physiological responses based on filtration rates, respiration and ammonia excretion between controls and groups receiving saline shocks. We found differences both in the individual effect of saline shocks and in the exposure to cadmium; we also found an important synergistic effect, which led us to conclude that the species response as biomonitor depends on the conditions of salinity and ecosystem dynamics. (C) 2016. Acad. Colomb. Cienc. Ex. Fis. Nat.
\end{abstract}

Key words: Biomonitoring; Cadmium; Isognomon alatus; Physiological responses.

\section{Introducción}

La contaminación marina en la costa Caribe colombiana proviene de múltiples fuentes, entre ellas el petróleo y sus derivados, diversos minerales, y contaminantes urbanos, industriales y agrícolas. El dragado remueve los sedimentos contaminados y hace que estos pasen a la columna de agua, donde son acumulados por organismos marinos como los bivalvos (Manjarréz, et al., 2008). Según los estudios del Instituto de Investigaciones Marinas y Costeras -
INVEMAR (Colombia), la contaminación por metales pesados se ha incrementado en las aguas del litoral caribe, siendo las zonas más afectadas aquellas cercanas a los principales asentamientos humanos como las ciudades de Cartagena, Barranquilla, Santa Marta, Coveñas, Tolú y Riohacha (INVEMAR, 2004).

\footnotetext{
*Correspondencia:

Juan Manuel Polo-Osorio,jmpoloo@unal.edu.co,juanchopolo-1@hotmail.com Recibido: 12 de mayo de 2016
}

Aceptado: 17 de octubre de 2016 
Las respuestas fisiológicas de las especies marinas y estuarinas, se ven afectadas directamente por la salinidad del medio, la cual se considera uno de los factores que más influye en su crecimiento y sobrevivencia, además de ser un factor ambiental que controla la distribución de las especies e influye en procesos fisiológicos como la tasa de filtración, respiración y excreción de amonio. Los estudios sobre la toxicidad de los metales pesados en organismos acuáticos en condiciones de laboratorio son importantes porque generan información que contribuye a predecir y a evaluar el estado de salud de los organismos, sus mecanismos de respuesta y los posibles efectos adversos sobre la biota de los ecosistemas acuáticos (Chung, 1978; Allen, et al., 1989; Lemus et al., 2012). Por lo tanto, es crucial entender las respuestas fisiológicas de un organismo ante una situación de estrés, ya que en medida en que es capaz de responder funcionalmente a los cambios en el ambiente, también debe hacerlo en el ecosistema. En este sentido, un acercamiento ecofisiológico permite entender la presencia de una especie en un lugar con determinadas características (Vernberg, 1983).

A pesar de que la diferencia de salinidad tiene implicaciones fisiológicas muy diversas, generalmente se piensa en los efectos osmóticos, ya que la capacidad de sobrevivir en diferentes medios se desarrolló mediante la evolución de un medio interno estable que actúa amortiguando los rigores del medio externo sobre los tejidos internos. Por ello, la capacidad de mantener un medio interno adecuado frente al estrés osmótico, el cual perturba el equilibrio iónico y osmótico del animal, ha tenido un papel muy importante en la evolución; de hecho, la distribución geográfica de los animales está determinada por los factores ambientales, entre los cuales la naturaleza osmótica del medio es uno de los más importantes (Morillo \& Belandria, 2006).

El efecto tóxico de los metales en su forma iónica se ha descrito en el cadmio, cuya toxicidad es muy compleja, ya que presenta múltiples posibilidades de formar macromoléculas, por ejemplo, reemplazando otros metales que desempeñan un papel muy importante en la actividad enzimática o reaccionan con grupos biológicos activos como los grupos carbosil, fenolosil, sulfidril, disulfito y fosfato (Theede \& Schoslz, 1982; Campos, 1987). Los metales presentan diferentes grados de afinidad con los diversos grupos químicos, y la gran afinidad del cadmio por el grupo sulfidril conduce a la formación, tanto en el hombre como en los animales, de proteínas de cadenas cortas con un alto contenido de cisteína, lo cual hace que su consumo, así sea incidental, sea altamente nocivo (Friberg, et al., 1976).

Isognomon alatus se considera una de las especies idóneas para el control en los estudios de bioacumulación de contaminantes y para valorar su respuesta frente a los cambios en los ambientes costeros debido a su resistencia a las variaciones de salinidad, temperatura y $\mathrm{pH}$, y por ser abundante en las áreas costeras del Caribe. Además, se trata de un organismo sésil, lo que, unido a su tolerancia a los metales pesados, facilita la monitorización (Campos, 1988; Ismail, 2006).
En este contexto, la presente investigación se propuso evaluar en el laboratorio el efecto combinado de la salinidad y la exposición al cadmio en las respuestas fisiológicas del bivalvo litoral I. alatus. Los resultados servirán como caso de estudio para una mejor comprensión de la relación entre los procesos ecofisiológicos normales y los patrones de distribución de las especies, así como las situaciones de estrés producidas por los cambios en la salinidad o por contaminantes como el cadmio. Asimismo, contribuirá a profundizar en el conocimiento del efecto de los factores ambientales en los procesos fisiológicos y a incentivar este tipo de investigaciones para la comprensión cabal de los mecanismos adaptativos que permiten a los organismos sobrevivir, crecer y reproducirse en medios con variaciones ambientales.

\section{Metodología}

Recolección y preparación de los organismos. El material biológico se obtuvo en La Escollera, sector de El Rodadero, cuyas coordenadas son $11^{\circ} 12^{\prime} 31,56^{\prime}$ 'N y $74^{\circ} 13^{\prime} 30,49^{\prime}$ 'O, en la región de Santa Marta, sitio donde se da en abundancia. Se recolectaron 200 individuos con una talla promedio de 4 a $6 \mathrm{~cm}$, los cuales se lavaron previamente para eliminar los epibiontes. Los animales se trasladaron al laboratorio del CECIMAR, en el Instituto de Investigaciones Marinas y Costeras - INVEMAR, donde se colocaron en acuarios de vidrio y se mantuvieron durante 30 días con aireación constante, dos recambios parciales de agua de mar diarios, y en tres salinidades diferentes (36, 27 y 18 UPS. Después del período de aclimatación, se hicieron los experimentos para determinar la respuesta de los individuos al choque salino. Para ello se dividieron las ostras de cada uno de los tres acuarios en tres grupos y tres réplicas, los cuales fueron expuestos a tres salinidades finales $(36,27,18)$, para un total de nueve experimentos: seis choques salinos y tres controles (Figura 1S, http://www.raccefyn.co/index.php/raccefyn/article/ downloadSuppFile/359/1414). Para determinar la respuesta metabólica de las ostras al choque salino, se midieron las tasas inicial y final de respiración, filtración y excreción de amonio. Después de este experimento sin cadmio, se realizó otro con cadmio, con el fin de determinar de qué manera este metal afectaba la respuesta metabólica de los organismos. En este segundo experimento se sometió a los individuos a una concentración no letal de $1 \mu \mathrm{g} / \mathrm{L}$ de cadmio, determinada con base en el estudio de Manjarréz, et al. (2008), quienes evaluaron la bioacumulación de metales pesados en I. alatus. El experimento completo se realizó por triplicado, con el fin de determinar si los resultados observados coincidían.

Experimento de medición de la tasa de consumo de oxígeno. Se midió la tasa respiratoria en cámaras respirométricas cerradas de flujo continuo (Hanke, et al., 1977). El suministro de agua se surtió mediante tres tanques de $19 \mathrm{~L}$ cada uno, con concentraciones de salinidad diferentes (36, $27,18)$. En cada cámara se introdujeron cinco ostras y luego se hizo fluir el agua durante 5 horas. La primera alícuota de agua se tomó en una botella Winkler de $50 \mathrm{ml}$ al cabo de la primera media hora del choque salino para esperar hasta que 
los individuos abrieran las valvas nuevamente. El oxígeno de la muestra se fijó con $0,5 \mathrm{ml}$ de sulfato de manganeso y $0,5 \mathrm{ml}$ de solución alcalina de yoduro. Posteriormente, se tomaron las muestras cada hora durante las siguientes 5 horas, y se las sometió al mismo tratamiento. La velocidad de flujo de la cámara se midió en cada tiempo con el fin de mantenerla constante, ya que no debía sobrepasar $20 \mathrm{ml} /$ min. Esto permitió observar las respuestas del consumo de oxígeno de los individuos en el tiempo frente a cambios bruscos de salinidad.

Para el segundo experimento se repitió el procedimiento anterior agregando cloruro de cadmio al tanque de suministro de agua de $19 \mathrm{~L}$ para obtener una concentración final de 0,387 mg de cadmio (Figura 2S, http://www.raccefyn.co/index.php/ raccefyn/article/downloadSuppFile/359/1416). La cantidad de oxígeno disuelto para cada muestra se determinó en el LABCAM de INVEMAR mediante el método de Winkler (1988) ajustado por Garay, et al. (2001). Para los cálculos del oxígeno consumido en los intervalos de tiempo, se tomó la diferencia de la concentración del oxígeno del agua entrante y saliente en $\mathrm{mg}, \mathrm{O}_{2} / \mathrm{L}$, y se la relacionó con la cantidad de agua que pasaba por la cámara en un tiempo dado, obteniendo el consumo de oxígeno en ml/L (Hanke, et al., 1977).

Tasa de filtración. La tasa de filtración se evaluó en cámaras estáticas de $2 \mathrm{~L}$, en las que se introdujeron cinco ostras de cada grupo por separado y con su respectivo control en agua de mar, con las salinidades propuestas y tres réplicas por tratamiento, adicionando, además, una suspensión de tinta china de $0,7 \mathrm{ml} / \mathrm{L}$. Después de la primera media hora se tomaron tres alícuotas de $10 \mathrm{ml}$ directamente de la cámara y así sucesivamente cada hora durante 5 horas, con el fin de determinar los cambios en el tiempo de las concentraciones de tinta china. La tasa de filtración se calculó con base en la diferencia de concentraciones de tinta china en cada intervalo de tiempo. Al igual que en el experimento anterior, los datos obtenidos se compararon con el grupo control en cada caso y con la salinidad de aclimatación. En el segundo experimento se repitió este procedimiento agregando $1 \mu \mathrm{g} / \mathrm{L}$ de cloruro de cadmio. La tasa de filtración (f) indica la cantidad de agua que pasa a través de las branquias del bivalvo en un tiempo determinado y se calcula en litros (L) por hora (h).

Tasa de excreción de amonio. Las muestras se tomaron de las cámaras estáticas utilizadas para la determinación de la tasa de filtración. La concentración de amonio excretada se determinó en el LAB-CAM del INVEMAR usando el método de azul de indofenol (INVEMAR, 2003); las muestras fueron tomadas directamente de las mismas cámaras que se usaron para la determinación de la tasa de filtración en los dos experimentos.

Análisis de los datos. Se calculó la tasa promedio de las respuestas y la respectiva desviación estándar a partir de los experimentos por triplicado. El efecto del choque salino se determinó mediante una prueba de Kruskal-Wallis para determinar las diferencias con respecto a la mediana, cuando las hipótesis son:
$\mathrm{H}_{0}=$ las distribuciones de las poblaciones son idénticas $\left(\alpha_{1}=\alpha_{2}=\alpha_{3}=\alpha_{4}=\alpha_{t}=0\right)$,

$\mathrm{H}_{\mathrm{a}}=$ al menos una de las distribuciones tiende a mostrar valores mayores que una de las otras poblaciones (algún $\alpha_{i} \neq 0$ ).

El nivel de significación $(\mathrm{H})$, se determinó usando la tabla de ji al cuadrado con grados de libertad n-1 (Daniels, 2005). El nivel de significación se determinó mediante comparaciones pareadas de los valores $\mathrm{T}_{\mathrm{a}}$ y $\mathrm{T}_{\mathrm{b}}$ calculados con respecto a $\mathrm{T}_{\text {Low }} \mathrm{y}_{\mathrm{Upper}}$ críticos de la tabla $\mathrm{T}$ de Wilcoxon. El efecto del cadmio se determinó mediante contrastes pareados utilizando una prueba de signos de Wilcoxon para muestras dependientes. El nivel de significación se determinó comparando el $\mathrm{T}$ calculado más pequeño con el $\mathrm{T}_{\text {Low }}$ de la tabla Wilcoxon para muestras independientes.

\section{Resultados}

Consumo de oxígeno. La prueba de Kruskall - Wallis $(\mathrm{H}$ calculada $>\mathrm{H}_{\text {critica }}(11,070) ; \alpha=0,05 ;$ gl 5) indicó diferencias en las tasas de consumo promedio del oxígeno frente a los cambios de salinidad y al efecto del cadmio en los tres experimentos con salinidades de aclimatación de $36\left(\mathrm{H}_{\text {calculada }}\right.$ 15,410), $27\left(\mathrm{H}_{\text {calculada }} 14,544\right)$ y $18\left(\mathrm{H}_{\text {calculada }} 11,573\right)$.

En los experimentos con salinidad de aclimatación de 36 , las pruebas en salinidades menores generaron una disminución en la tasa de consumo de oxígeno. También se observó una disminución en el consumo de oxígeno cuando los individuos fueron sometidos al choque salino de $36: 18$; no obstante, en el choque salino de 36:27 se observó un incremento evidente en el consumo con respecto al control, siendo esta la respuesta en la cual fue más evidente la actividad respiratoria (Figura 1, Tabla 1).

En la salinidad de aclimatación de 27, también se observó que el cadmio reducía drásticamente el consumo de oxígeno y que este efecto era independiente del choque salino. En los experimentos sin cadmio, el efecto del choque salino generó un descenso leve en la tasa de consumo promedio de oxígeno con respecto al control, evidenciando una respuesta similar a la del experimento de aclimatación de 36. No obstante, las respuestas de los individuos aclimatados a una salinidad de 18 presentaron un comportamiento diferente a los otros experimentos, ya que frente al efecto del cadmio, el choque salino de 18:27 presentó un notable incremento en el consumo, excediendo incluso la tasa de consumo del control, la cual es una respuesta atípica frente a la exposición al cadmio. Los choques salinos de los experimentos sin cadmio evidenciaron un incremento leve en el consumo de oxígeno, acorde con lo observado en los otros experimentos (Figura 3).

Tasa de filtración. Se observaron diferencias en las tasas de filtración entre los tres tratamientos $(\mathrm{K}-\mathrm{W})=\mathrm{p}$-valor $<$ 0,05. En la salinidad de acondicionamiento de 36, se observó que la tasa de filtración disminuía en todos los casos frente a la tasa de control, tanto por el efecto del cadmio como por el choque salino, siendo mayor el descenso frente al cadmio en el choque de Cd 36:18; se observó, además, un efecto sinérgico entre ambas variables. Cuando la salinidad de acondicionamiento fue de 27 , se observó un descenso 

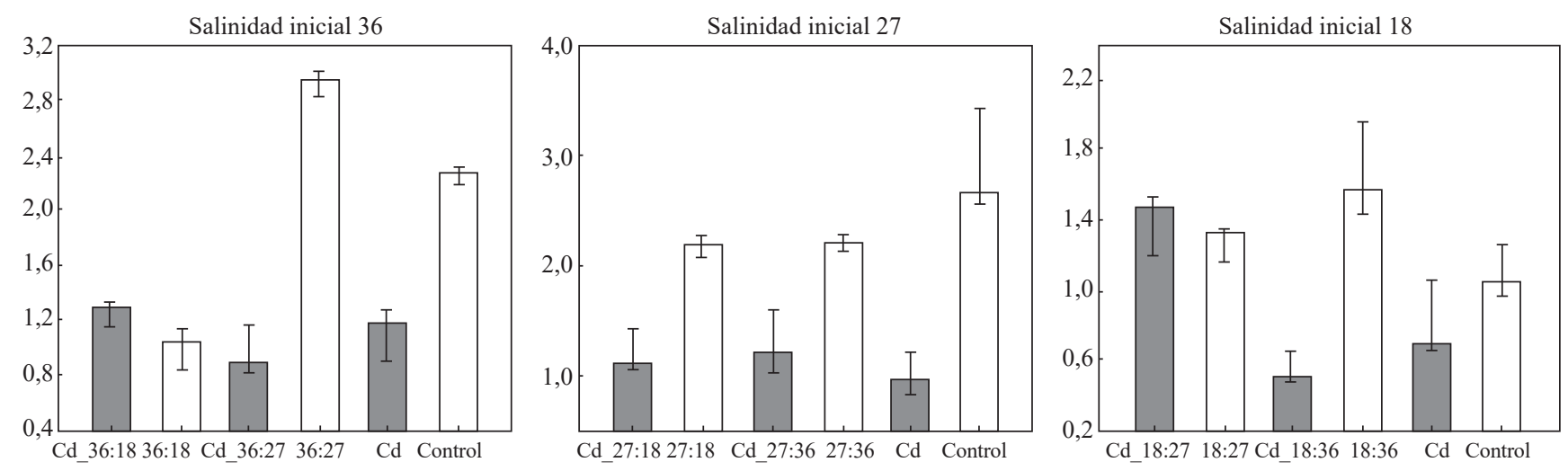

Figura 1. Tasas de consumo medio de oxígeno en los tres experimentos con salinidades de acondicionamiento de 36,27 y 18 , con base en la media geométrica. La variación de las réplicas se representó mediante el error estándar.

\begin{tabular}{|c|c|c|c|c|c|c|c|c|c|c|c|c|c|c|}
\hline \multicolumn{5}{|c|}{ Salinidad 36} & \multicolumn{5}{|c|}{ Salinidad 27} & \multicolumn{5}{|c|}{ Salinidad 18} \\
\hline \multicolumn{5}{|c|}{ Choque salino } & \multicolumn{5}{|c|}{ Choque salino } & \multicolumn{5}{|c|}{ Choque salino } \\
\hline $\mathrm{T}<; \mathrm{T}>$ & Control & & $\mathrm{T}<; \mathrm{T}>$ & $\mathrm{Cd}$ & $\mathrm{T}<; \mathrm{T}>$ & Control & & $\mathrm{T}<; \mathrm{T}>$ & $\mathrm{Cd}$ & $\mathrm{T}<; \mathrm{T}>$ & Control & & $\mathrm{T}<; \mathrm{T}>$ & $\mathrm{Cd}$ \\
\hline S 36:18 & $6 ; 15^{*}$ & & Cd_36:18 & $10 ; 11$ & S 27:18 & $6 ; 15^{*}$ & & Cd_27:18 & $8 ; 13$ & S 18:36 & $6 ; 15^{*}$ & & Cd_18:36 & $7 ; 13,5$ \\
\hline S 36:27 & $6 ; 15^{*}$ & & Cd_36:27 & $10 ; 11$ & S 27:36 & $6 ; 15^{*}$ & & Cd_27:36 & $9 ; 12$ & S 18:27 & 9,$5 ; 11,5$ & & Cd_18:27 & $7 ; 14$ \\
\hline $\mathrm{T}<; \mathrm{T}>$ & S 36:27 & & $\mathrm{T}<; \mathrm{T}>$ & Cd_36:27 & $\mathrm{T}<; \mathrm{T}>$ & S 27:36 & & $\mathrm{T}<; \mathrm{T}>$ & Cd_36:27 & $\mathrm{T}<; \mathrm{T}>$ & S 18:27 & & $\mathrm{T}<; \mathrm{T}>$ & Cd_36:27 \\
\hline \multirow[t]{6}{*}{ S 36:18 } & $6 ; 15^{*}$ & & Cd 36:18 & $8 ; 13$ & S 27:18 & $10 ; 11$ & & Cd_27:18 & $6 ; 15^{*}$ & S 18:36 & $6 / 15^{*}$ & & \begin{tabular}{|l|}
$\mathrm{Cd} 18: 36$ \\
\end{tabular} & $6 ; 15^{*}$ \\
\hline & \multicolumn{4}{|c|}{ Efecto combinado } & & \multicolumn{4}{|c|}{ Efecto combinado } & & \multicolumn{4}{|c|}{ Efecto combinado } \\
\hline & $\mathrm{T}$ & Control & S 36:18 & S 36:27 & & $\mathrm{T}$ & Control & S 27:18 & S 27:36 & & $\mathrm{T}$ & Control & \begin{tabular}{|l|} 
S 18:36 \\
\end{tabular} & S 18:27 \\
\hline & $\mathrm{Cd}$ & $0^{*}$ & - & - & & $\mathrm{Cd}$ & $0^{*}$ & - & - & & $\mathrm{Cd}$ & $2 *$ & - & - \\
\hline & Cd_36:18 & $0^{*}$ & $2 *$ & - & & Cd_27:18 & $0^{*}$ & $0^{*}$ & - & & \begin{tabular}{|l|} 
Cd_18:36 \\
\end{tabular} & $0^{*}$ & $0^{*}$ & - \\
\hline & Cd_36:27 & $0^{*}$ & - & $0^{*}$ & & Cd_27:36 & $0^{*}$ & - & $0^{*}$ & & Cd_18:27 & $1^{*}$ & - & $2 *$ \\
\hline
\end{tabular}

Tabla 1. Niveles de significación de los choques salinos mediante pruebas de Wilcoxon para muestras independientes $\left(\alpha=0,05 ; T_{10 w}\right.$ $>6 \mathrm{y} \mathrm{T}_{\text {upper }}<15$ ); el efecto combinado del cadmio y el choque salino, se contrastó mediante una prueba de Wilcoxon para muestras dependientes $\left(\alpha=0,05 ; \mathrm{T}_{\text {calculado }}>2\right)$.

drástico en la capacidad filtradora frente al cadmio, en especial en el incremento de Cd 27:36. Sin embargo, el choque salino en este caso pareció generar una respuesta leve en la capacidad de filtración de las ostras. En la salinidad de acondicionamiento de 18 se observó un comportamiento muy similar a los experimentos con acondicionamiento a una salinidad de 36; la mayor tasa de filtración se observó en el choque de 18:36 y el efecto del cadmio causó un descenso drástico en la tasa de filtración, en especial en el choque de 18:27 (Figura 2, Tabla 2).

Tasa de excreción de amonio. Se determinaron diferencias en las tasas de excreción media de amonio en los tres tratamientos $((\mathrm{K}-\mathrm{W})=\mathrm{p}$-valor $<0,05)$. En la salinidad de aclimatación de 36, la tasa de excreción se elevó notablemente cuando los individuos se expusieron al cadmio, presentando, además, un efecto sinérgico con el choque salino, en el cual la mayor tasa de excreción se observó en el experimento de $\mathrm{Cd}$ 36:18. En los experimentos sin cadmio el efecto del choque fue inverso al observado frente al cadmio, y el experimento de 36:18 presentó la menor tasa de excreción (Tabla 3).
Los experimentos de acondicionamiento de 27 presentaron incrementos significativos en la tasa de excreción de amonio, excepto en el descenso de Cd 27:18, en el cual la tasa de excreción disminuyó más allá de los límites del control y presentó el menor efecto de todos los grupos; sin embargo, el efecto del choque salino en los experimentos sin cadmio al parecer no tuvo un efecto significativo sobre la capacidad de excreción de los organismos. En la salinidad de acondicionamiento de 18 , se observó también una tasa mayor de excreción frente al cadmio, así como un efecto sinérgico con el choque salino, siendo mayor en el experimento de Cd 18:27; el efecto del choque salino presentó la misma tendencia que la del efecto observado bajo los efectos del cadmio, pero menos intenso (Figura 3).

\section{Discusión}

Consumo de oxígeno. La exposición al cadmio produjo una disminución en las tasas de consumo de oxígeno en la tres salinidades de aclimatación, y se observó que I. alatus respondía cerrando completamente sus valvas, respuesta que también se presentó, pero de forma independiente, con el 

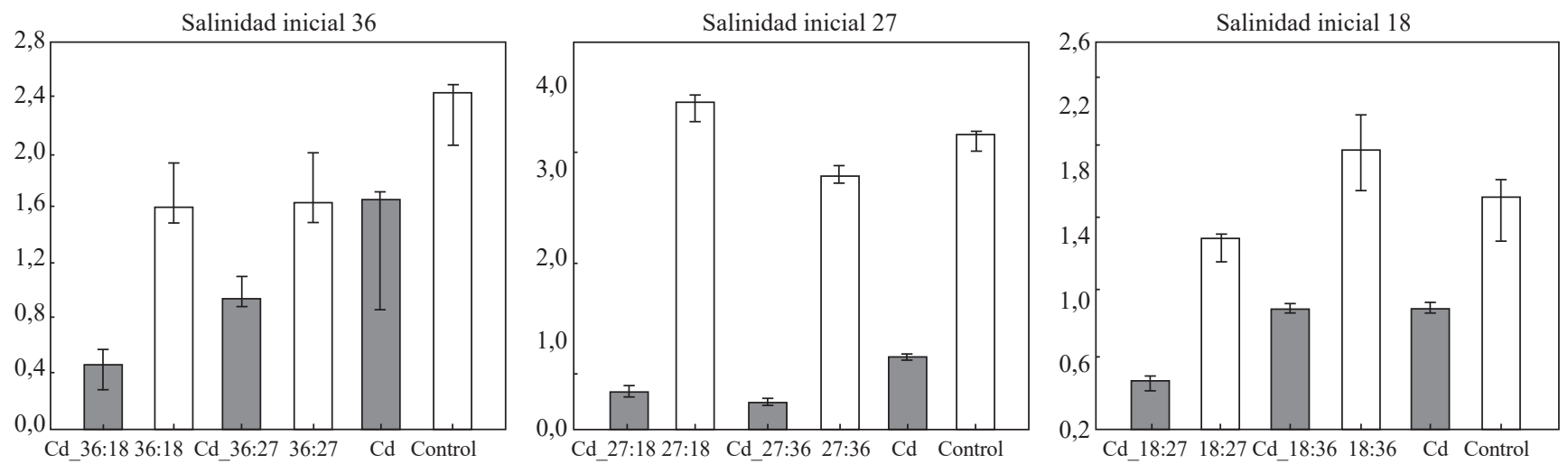

Figura 2. Tasas de filtración media en los tres experimentos con salinidades de acondicionamiento de 36 , 27 y 18 , con base en la media geométrica. La variación de las réplicas se representó mediante el error estándar.

\begin{tabular}{|c|c|c|c|c|c|c|c|c|c|c|c|c|c|c|}
\hline \multicolumn{5}{|c|}{ Salinidad 36} & \multicolumn{5}{|c|}{ Salinidad 27} & \multicolumn{5}{|c|}{ Salinidad 18} \\
\hline \multicolumn{5}{|c|}{ Choque salino } & \multicolumn{5}{|c|}{ Choque salino } & \multicolumn{5}{|c|}{ Choque salino } \\
\hline $\mathrm{T}<; \mathrm{T}>$ & Control & & $\mathrm{T}<; \mathrm{T}>$ & $\mathrm{Cd}$ & $\mathrm{T}<; \mathrm{T}>$ & Control & & $\mathrm{T}<; \mathrm{T}>$ & $\mathrm{Cd}$ & $\mathrm{T}<; \mathrm{T}>$ & Control & & $\mathrm{T}<; \mathrm{T}>$ & $\mathrm{Cd}$ \\
\hline S 36:18 & $6 ; 15^{*}$ & & Cd_36:18 & $6 ; 15^{*}$ & S 27:18 & $7 ; 14$ & & Cd_27:18 & $6 ; 15^{*}$ & S 18:36 & $6 ; 15^{*}$ & & Cd_18:36 & $8 ; 15^{*}$ \\
\hline S 36:27 & $6 ; 15^{*}$ & & Cd_36:27 & $6 ; 15^{*}$ & S $27: 36$ & 6,$5 ; 14,5^{*}$ & & Cd_27:36 & $6 ; 15^{*}$ & S 18:27 & 6,$5 ; 14,5^{*}$ & & Cd_18:27 & $6 ; 14,5^{*}$ \\
\hline \multirow{7}{*}{$\begin{array}{l}\mathrm{T}<; \mathrm{T}> \\
\mathrm{S} 36: 18\end{array}$} & S 36:27 & & $\mathrm{T}<; \mathrm{T}>$ & Cd_36:27 & $\mathrm{T}<; \mathrm{T}>$ & S 36:27 & & $\mathrm{T}<; \mathrm{T}>$ & Cd_36:27 & $\mathrm{T}<; \mathrm{T}>$ & S 18:27 & & $\mathrm{T}<; \mathrm{T}>$ & Cd_18:27 \\
\hline & 6,$5 ; 14,5$ & & Cd 36:18 & $6 ; 15^{*}$ & S 27:18 & $6 ; 15^{*}$ & & Cd 27:18 & $6 ; 15^{*}$ & S $18: 36$ & $6 ; 15^{*}$ & & Cd_18:36 & $6 ; 15^{*}$ \\
\hline & \multicolumn{4}{|c|}{ Efecto combinado } & & \multicolumn{4}{|c|}{ Efecto combinado } & & \multicolumn{4}{|c|}{ Efecto combinado } \\
\hline & $\mathrm{T}$ & Control & S 36:18 & S 36:27 & & $\mathrm{T}$ & Control & S 27:18 & S 27:36 & & $\mathrm{T}$ & Control & S $18: 36$ & S 18:27 \\
\hline & $\mathrm{Cd}$ & $0^{*}$ & - & - & & $\mathrm{Cd}$ & $0^{*}$ & - & - & & $\mathrm{Cd}$ & $0^{*}$ & - & - \\
\hline & Cd_36:18 & $0^{*}$ & $0^{*}$ & - & & Cd_27:18 & $0^{*}$ & $0^{*}$ & - & & Cd_18:36 & $0^{*}$ & $0^{*}$ & - \\
\hline & Cd_36:27 & $0^{*}$ & - & 0* & & Cd_27:36 & $0^{*}$ & - & 0* & & Cd_18:27 & 0* & - & $0^{*}$ \\
\hline
\end{tabular}

Tabla 2. Niveles de significación de los choques salinos mediante pruebas de Wilcoxon para muestras independientes $\left(\alpha=0,05 ; \mathrm{T}_{\text {low }}\right.$ $>6 \mathrm{y} \mathrm{T}_{\text {upper }}<15$ ); el efecto combinado del cadmio y el choque salino, se contrastó mediante una prueba de Wilcoxon para muestras dependientes $\left(\alpha=0,05 ; \mathrm{T}_{\text {calculado }}>2\right)$.

cambio de salinidad. Mohan, et al. (1986), encontraron una respuesta metabólica similar a la observada en este estudio en Perna viridis, cuyo consumo de oxígeno descendía al ser expuesta a concentraciones de 0,04 y 0,05 ppm de cadmio y mercurio, respuesta que también se observó frente a los cambios de salinidad. Por consiguiente, la disminución en el consumo de oxígeno en I. alatus puede estar asociada a procesos de anaerobiosis por el cerramiento de la valvas, los cuales, según Salanki $(1965,1968)$, son típicos de los bivalvos bajo condiciones desfavorables como una respuesta mecánica que resulta en una diminución del oxígeno.

La ostra I. alatus es una especie osmoconforme, que cambia su concentración interna de acuerdo al medio externo, lo cual exige un esfuerzo y la obliga a responder tanto mecánica como fisiológicamente; en este caso, las ostras disminuyen el consumo de oxígeno por el cerramiento de la valvas, pero también pueden darse procesos de anaerobiosis para mantener una baja actividad metabólica y compensar, así, los cambios ambientales. Según Chandran
(2002), estas respuestas pueden estar influenciadas por factores específicos como el tamaño, la alimentación, las condiciones ambientales, el estado fisiológico del animal, la salinidad u otros tipos de estrés ambiental.

Al observar la respuesta metabólica al choque salino de 36 a 27 , la tasa de consumo de oxígeno fue mucho mayor, por encima de la aclimatación del control. Al contrario, al observar un choque salino mucho mayor, de 36 a 18 , el consumo de oxígeno fue menor, lo cual indicaría que a medida que desciende la salinidad en el medio, los procesos de adaptación a estas condiciones pueden ser muy lentos.

Como se observa en la Figura 3, en salinidades de 27, las tasas de consumo de oxígeno de I. alatus disminuyeron, independientemente de si el choque salino respondía a un incremento de la salinidad a 36 o a un descenso a 18 . En salinidades inferiores hubo un efecto sinérgico entre el choque salino a 18 y la exposición al cadmio, como se observa en la figura 3. Según Lewis (1990), en los bivalvos se da un proceso fisiológico similar frente al exceso de cobre: 


\begin{tabular}{|c|c|c|c|c|c|c|c|c|c|c|c|c|c|c|}
\hline \multicolumn{5}{|c|}{ Salinidad 36} & \multicolumn{5}{|c|}{ Salinidad 27} & \multicolumn{5}{|c|}{ Salinidad 18} \\
\hline \multicolumn{5}{|c|}{ Choque salino } & \multicolumn{5}{|c|}{ Choque salino } & \multicolumn{5}{|c|}{ Choque salino } \\
\hline $\mathrm{T}<; \mathrm{T}>$ & Control & & $\mathrm{T}<; \mathrm{T}>$ & $\mathrm{Cd}$ & $\mathrm{T}<; \mathrm{T}>$ & Control & & $\mathrm{T}<; \mathrm{T}>$ & $\mathrm{Cd}$ & $\mathrm{T}<; \mathrm{T}>$ & Control & & $\mathrm{T}<; \mathrm{T}>$ & $\mathrm{Cd}$ \\
\hline S 36:18 & $6 ; 15^{*}$ & & Cd_36:18 & $6 ; 15^{*}$ & S 27:18 & $8 ; 14$ & & Cd_27:18 & $6 ; 15^{*}$ & S 18:36 & $6 ; 15^{*}$ & & Cd_18:36 & $6 ; 15^{*}$ \\
\hline S 36:27 & $6 ; 15^{*}$ & & Cd_36:27 & $6 ; 15^{*}$ & \begin{tabular}{|l|} 
S 27:36 \\
\end{tabular} & 6,$5 ; 14,5$ & & Cd_27:36 & $6 ; 15^{*}$ & \begin{tabular}{|l|} 
S 18:27 \\
\end{tabular} & $6 ; 15^{*}$ & & Cd_18:27 & $6 ; 15^{*}$ \\
\hline $\mathrm{T}<; \mathrm{T}>$ & S 36:27 & & $\mathrm{T}<; \mathrm{T}>$ & Cd_36:27 & $\mathrm{T}<; \mathrm{T}>$ & S 27:36 & & $\mathrm{T}<; \mathrm{T}>$ & Cd_36:27 & $\mathrm{T}<; \mathrm{T}>$ & S 18:27 & & $\mathrm{T}<; \mathrm{T}>$ & Cd 36:27 \\
\hline \multirow[t]{6}{*}{ S 36:18 } & $6 ; 15^{*}$ & & Cd 36:18 & $6 ; 15^{*}$ & S 27:18 & $9 ; 12$ & & Cd_27:18 & $6 ; 15^{*}$ & \begin{tabular}{|l|} 
S $18: 36$ \\
\end{tabular} & $10 ; 11$ & & Cd 18:36 & $6 ; 15^{*}$ \\
\hline & \multicolumn{4}{|c|}{ Efecto combinado } & & \multicolumn{4}{|c|}{ Efecto combinado } & & \multicolumn{4}{|c|}{ Efecto combinado } \\
\hline & $\mathrm{T}$ & Control & S 36:18 & S 36:27 & & $\mathrm{T}$ & Control & S 27:18 & S 27:36 & & $\mathrm{T}$ & Control & S 18:36 & S 18:27 \\
\hline & $\mathrm{Cd}$ & $0^{*}$ & & & & $\mathrm{Cd}$ & $0 *$ & & & & $\mathrm{Cd}$ & $0^{*}$ & & \\
\hline & Cd_36:18 & $0^{*}$ & $0 *$ & & & Cd_27:18 & $0^{*}$ & $0 *$ & & & \begin{tabular}{|l|} 
Cd_18:36 \\
\end{tabular} & $0^{*}$ & $0 *$ & \\
\hline & Cd_36:27 & $0^{*}$ & - & $0^{*}$ & & Cd_27:36 & $0^{*}$ & - & $0^{*}$ & & Cd_18:27 & $0^{*}$ & - & $0^{*}$ \\
\hline
\end{tabular}

Tabla 3. Niveles de significación de los choques salinos mediante pruebas de Wilcoxon para muestras independientes $\left(\alpha=0,05 ; \mathrm{T}_{\text {low }}\right.$ $\left.>6 \mathrm{y} \mathrm{T}_{\text {upper }}<15\right)$; el efecto combinado del cadmio y el choque salino se contrastó mediante una prueba de Wilcoxon para muestras dependientes $\left(\alpha=0,05 ; \mathrm{T}_{\text {calculado }}>2\right)$.
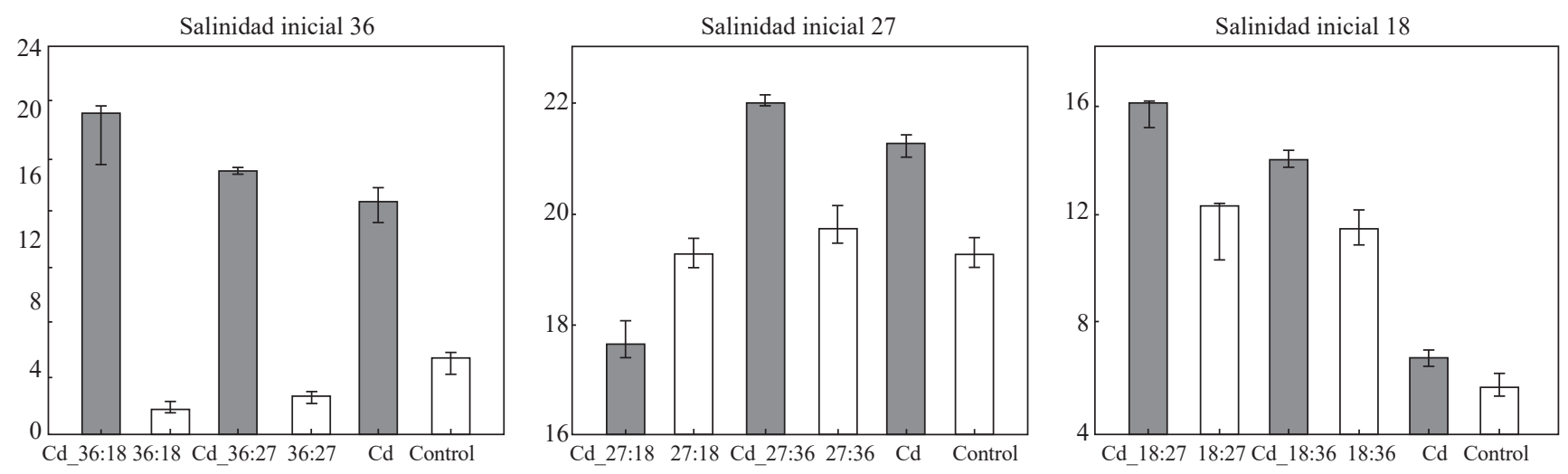

Figura 3. Tasas de excreción media de amonio en los tres experimentos con salinidades de acondicionamiento de 36, 27 y 18 , con base en la media geométrica; la variación de las réplicas se representó mediante el error estándar.

disminuye la tasa de captación de oxígeno y se reduce la tasa de filtración debido a una menor actividad ciliar en respuesta al incremento en los niveles de contaminantes (Almeida, et al., 2003).

Tasa de filtración. Al observar las respuestas de las tasas de filtración en los organismos aclimatados a una salinidad de 36 (Figura 4), se determinó que las tasas disminuyeron en todos los casos frente a la tasa de control, tanto por el efecto del cadmio como por el choque salino, y que el descenso fue mayor frente al cadmio en el choque de $\mathrm{Cd}$ 36:18, observándose un efecto sinérgico entre ambas variables. Según Chandran (2002), el cierre de las valvas es uno de los mecanismos que adoptan estos organismos para responder a los desequilibrios del medio ambiente exterior; además, ayuda a una especie determinada a resistir, al menos temporalmente, un cambio drástico en la concentración del medio externo. Al cerrar las valvas en respuesta a algún factor estresante del medio, los bivalvos modifican su proceso respiratorio y cambian a la anaerobiosis, con un incremento del ácido láctico en la hemolinfa y un cambio en la acidez del líquido intervalvar (Baqueiro, et al., 2007), lo cual probablemente ocurrió en los experimentos en los que los organismos se aclimataron a salinidades de 27 y 18 , y se sometieron después a una salinidad de 36 , lo cual causó un descenso en la tasa de filtración. Se ha demostrado que un proceso fisiológico similar se produce en el mejillón cebra Dreissena polymorpha, con la disminución en la tasa de captación y en el consumo de oxígeno, así como en las tasas de filtración por exceso de cobre en el ambiente (Lewis, 1990). La tasa de filtración está asociada con una actividad ciliar menor en respuesta al incremento en los niveles de contaminantes (Almeida, et al., 2003). Una reducción de este tipo puede explicarse por el endurecimiento de las estructuras filtradoras debido a la exposición a condiciones extremas; en el caso del estudio de Baqueiro, et al., (2007), los cambios repentinos de la salinidad ejercieron un efecto constante sobre las poblaciones, lo cual coincide con lo ocurrido en este trabajo, ya que las ostras se aclimataron en diferentes salinidades y se expusieron de forma constante a salinidades altas (en este caso de 36), lo cual dio lugar a cambios irreversibles que causaron la mayor parte de las variaciones. 
Tasa de excreción de amonio. Como se observa en la figura 5, con el descenso de la salinidad de aclimatación de 36 a 27 y 18, la tasa de excreción de amonio en I. alatus fue mucho más baja que la del control, lo cual indicaría que frente al choque salino, las ostras no excretan iones de forma significativa a través de los órganos correspondientes, pues ello solo ocurre si el animal posee diversos mecanismos para hacer frente al estrés osmótico (mecanismos como el cierre de las valvas o mecanismos fisiológicos). Las ostras acondicionadas a salinidades de 27 y 18 presentaron tasas de excreción de amonio mayores que las de sus respectivos controles, lo cual indica que eliminan iones activamente y permanecen en un estado osmoconforme e isotónico con respecto al medio, lo que podría causar que el agua traspase rápidamente las paredes del cuerpo de los organismos; es evidente, entonces, que las ostras acondicionadas a bajas salinidades difícilmente retornan a una condición estable luego de permanecer por mucho tiempo sometidas a una salinidad baja. Según Chandran (2002), el cierre prolongado de las valvas puede disparar la osmolaridad de los tejidos, desencadenando la anaerobiosis y la acumulación de productos de desecho en el interior; de ahí que el cierre de las valvas no sea una solución permanente para evadir los cambios de salinidad.

Los individuos de I. alatus acondicionados en una salinidad de 36 presentaron poca variación en la tasa de excreción, ya que en un medio más diluido de iones deben generar una excreción más diluida, con el fin de conservar su concentración iónica interna. En una salinidad de 27, el medio tiene una menor concentración de iones, así que para mantener el estado isotónico, I. alatus aumenta su tasa de excreción de amonio. Según Navarro \& González (1998), este aumento se debe al metabolismo de las proteínas como fuente de energía para contrarrestar la demanda debida a la adaptación; por ello, en este experimento, las ostras empezaron a regular activamente la presión osmótica del medio (osmorregulación). En la salinidad de 18 perdieron la capacidad de eliminar iones a través de la orina y eliminaron una mayor proporción de iones para equilibrar el medio, lo cual hacen, probablemente, desdoblando proteínas, como ya se mencionó. Estos resultados coinciden con los de Navarro \& González (1998), quienes en su estudio sobre el efecto de la salinidad en la excreción de amonio en Argopecten purpuratus, determinaron que esta especie aumentó su tasa de excreción de amonio frente a una disminución de la salinidad de 30 a $24 \%$, lo cual demuestra su capacidad para regular el volumen mediante el rompimiento y utilización de los aminoácidos como reguladores isosmóticos intracelulares a medida que la salinidad disminuye. Los resultados del presente estudio también se ven apoyados por lo reportado por Livingstone, et al., (1979), quienes determinaron un aumento de la tasa de excreción de amonio en el bivalvo Mytilus edulis asociado con el aumento de la concentración de aminoácidos en la hemolinfa a medida que disminuía la salinidad. En los experimentos con cadmio se determinó que las ostras presentaron tasas de excreción de amonio mayores que en el experimento sin cadmio. Con la exposición al cadmio las tasas aumentaron debido a que aumentó el estrés de las ostras y de tensores en el medio. Chandran (2002) ha señalado que todas las especies eurihalinas de bivalvos, como es el caso de I. alatus, conservan un entorno ambiental en la cavidad del manto durante algún tiempo cuando la fluctuación de la salinidad en el medio ambiente es mayor que el límite de tolerancia; cuando el animal se ve obligado a vivir en la zona de resistencia, la velocidad con la que regresa al nuevo equilibrio después de la exposición es un indicativo de la perfección de la adaptación.

Como se determinó en esta investigación, el cadmio tuvo un gran efecto en las respuestas fisiológicas de $I$. alatus, ya que redujo casi en su totalidad la capacidad de filtrar el material suspendido en la columna de agua, además de disminuir la tasa de consumo de oxígeno y aumentar la de excreción de amonio. Esto indica que hubo un efecto sinérgico del choque salino y la presencia de cadmio, aunque el choque salino fue más evidente, como ya se mencionó. Por otro lado, se observó que el cadmio pudo haber afectado los componentes celulares de I. alatus y, así, disminuir el metabolismo mitocondrial provocando un desequilibrio energético en estos organismos (Sokolova, $\boldsymbol{e t}$ al., 2005), lo cual resultó en una disminución generalizada del metabolismo celular (Ciocan \& Rotchell, 2004).

La contaminación del medio ambiente con metales pesados, en este caso el cadmio, es un problema serio a nivel mundial, ya que este es probablemente el elemento más biotóxico y uno de los contaminantes más importantes debido a los efectos que produce sobre la biota marina (Sadiq, 1992).

\section{Conclusiones}

Se determinó que existen efectos sinérgicos entre los choques salinos y la exposición al cadmio en las respuestas fisiológicas de I. alatus. Los individuos respondieron mecánicamente con el cierre de las valvas y fisiológicamente iniciando procesos de anaerobiosis frente a la exposición al cadmio. La tasa de excreción aumentó con la exposición a este metal cadmio.

\section{Información suplementaria}

Figura 1S. Montaje de los experimentos para la medición de la tasas de filtración y excreción de amonio. Vea la figura $1 \mathrm{~S}$ en: http://www.raccefyn.co/index.php/raccefyn/article/download SuppFile/359/1414

Figura 2S. Montaje del experimento para la medición de la tasa de consumo de oxígeno. Se observan los tanques de agua con cada una de las salinidades establecidas y las cámaras respirométricas cerradas de flujo continuo en las cuales se colocaron las ostras. Vea la figura $2 \mathrm{~S}$ en: http://www.raccefyn.co/index.php/raccefyn/ article/downloadSuppFile/359/1416 


\section{Agradecimientos}

Esta investigación se realizó con el apoyo del CECIMAR, de la Sede Caribe de la Universidad Nacional de Colombia y el INVEMAR a través del programa de Calidad Ambiental Marina (CAM), a los que les expresamos nuestros agradecimientos, como la tesis de maestría de uno de los autores (JMP).

Contribución No. 444 del Instituto de Estudios en Ciencias del Mar, CECIMAR, de la sede Caribe de la Universidad Nacional de Colombia.

\section{Conflicto de intereses}

Los autores manifiestan que no tienen conflicto de intereses.

\section{Referencias}

Akberali, H.B \& Trueman, E.R. 1985. Effects of environmental stress on marine bivalve molluscs. En Advances in Marine Biology, Blaxter J.H.S. Russel, F.S. \& Younge, M (eds). Academic Press, New York. 22: 101-198.

Almeida, E. A., Bainy, A.C.D, Medeiros, M.H.G, Di Mascio, P. 2003. Effects of trace metal and exposure to air on serotonin and dopamine levels in tissues of the mussel Perna perna. Marine Pollution Bulletin. 46: 1485-1490.

Allen, S.E., Parkinson, J.A, Rowland, A.P. 1989. Pollutants. En: O. Mead (editores). Chemical Analysis of Ecological materials. Blackwell Publications, Oxford, England. p. 201-239.

Baqueiro, C.E., Aldana-Aranda, D., Sevilla, M.L., RodríguezEspinosa, P.F. 2007. Variations of gametogenic and spawning patterns of the oyster Crassostrea virginica (Gmelin, 1971). Pueblo Viejo Lagoon, Veracruz, México. Transitional Waters Bulletin. 2: 37-46.

Campos, N.H. 1987. Los metales pesados, su contaminación y sus efectos tóxicos. Revista de Contaminación Ambiental. 9 (17): 63-70.

Campos, N.H. 1988. Selected bivalves for monitoring of heavy metal contamination in the Colombian Caribbean. Springer Berlin Heidelberg. p. 270-275.

Chandran, V. 2002. Intracellular osmoregulation in the estuarine mollusc Villorita cyprinoides var. cochinensis (Mollusca: Bivalvia) Hanley. Doctoral dissertation. Department of Marine Biology, Microbiology and Biochemistry. Faculty of Marine Sciences. Kochi, India.

Chung, K.S. 1978. Cadmium tolerance of the white mullet Mugil curema and its use to predict survival, probability in polluted sea waters. Bol. Inst. Oceanogr. Oriente. 17 (1-2): 105-107.

Ciocan, C.M. \& Rotchel, J.M.l. 2004. Cadmium induction of metallothionein isoforms in juvenile and adult mussel Mytilus edulis. Environ. Sci. Tech. 38: 1073-1078.

Daniels, W. W. 2005. Bioestadística. Base para las ciencias de la salud. Editorial Limusa, S.A. de C.V. Grupo Noriega Editores. México, D.F. p. 141.

Friberg, L., Kjellstrom, T., Nordberg, G.F. 1986. Cadmium. Handbook on the toxicology of metals, 2nd edition, Elsevier Science Publishers, Amsterdam, New York. P 342.

Garay, J.A., Marín, B., Calvano, N., Ramírez, G., Troncoso, W., Medina, O.L. 2001. Diagnóstico y evaluación de la calidad ambiental marina en el Caribe y Pacífico colombiano. Red de vigilancia para la protección y conservación de la calidad de las aguas marinas y costeras. Tomo II. Informe Final. INVEMAR. p 82.

Hanke, W., Hamdorf, K., Horn, E., Schlieper, C. 1977. Praktikum der Zoophysiologie. Gustav Fisher Verlag, Stuttgart. 350.
INVEMAR. 2003. Manual de técnicas analíticas para la determinación de parámetros físico químicos y contaminantes marinos. (Aguas, sedimentos y organismos). Instituto de Investigaciones Marinas y Costeras. Programa de Calidad Ambiental Marina (CAM). p. 40

INVEMAR. 2004. Programa nacional de investigación, evaluación, prevención, reducción y control de fuentes terrestres y marinas de contaminación marina. En prensa.

Ismail, A. 2006. The use of intertidal mollusks in the monitoring of heavy metals and organotin compounds in the west coast of Peninsular Malaysia. Coastal and Marine Science. 30 (1): 401-406.

Lemus, M., Marín, L., Aponte, Chung, K. 2012. Metalotioninas, glutación y consumo de oxígeno en el bivalvo Perna viridis expuesto al cadmio. Revista Científica, FCV-LUZ. XXII, 4: 376-382.

Lewis, A. G. 1990. The biological importance of copper. A literature review. Final report INCA project No. 223. Disponible en: http://scirus.landingzone.nl/other/?q=Type $\% 20$ your $\% 20$ own $\% 20$ search $\% 20$ here.

Livingstone, D.R., Widdows, J., Fieth, P. 1979. Aspects of nitrogen metabolism of the common mussel Mytilus edulis: Adaptation to abrupt and fluctuating changes in salinity. Marine Biology. 53: 41-55.

Manjarréz, G., Castro-Angulo, I., Utría-Padilla, L. 2008. Bioacumulación de cadmio en ostras de la bahía de Cartagena. Revista Ingenierías Universidad de Medellín. 7 (13): 11-20.

Matsushima, O., Katayama, H., Yamada, K. 1987. The capacity of intracellular osmoregulation mediated by free amino acids in three bivalve molluscs. Journal of Experimental Marine Biology and Ecology. 109: 93-99.

Mohan C.V., Gupta T.R.C., Shetty H.P.C., Menon N.R. 1986. Combined toxicity of mercury and cadmium to the tropical green mussel Perna viridis. Diseases of aquatic organisms. 2: $65-72$.

Morillo, N. \& Belandria, J.C. (2006). Utilización de moluscos bivalvos para el tratamiento de efluentes en granjas camaroneras. Serie: INIA Divulga (Venezuela). 8: 47-50.

Navarro, J.M. \& González C.M. (1998). Physiological responses of the Chilean scallop Argopecten purpuratus to decreasing salinities. Aquaculture. 167: 315-327.

Sadiq, M. 1992. Heavy metals. En: Toxic metal chemistry in marine environments. Marcel Dekker, Inc. New York, 390 p.

Salanki, J. 1965. Oxygen level as a specific regulator of the rhythrmc activity of freshwater mussel Anodonta cygenea. Acta biologica Academiae Scientiarum Hungaricae. 15: 299-310.

Salanki, J. 1968. Role of cerebral ganglia in the regulation of activity in freshwater mussel Anodonta cygnea. En Salanlu, J. (editor). Neurobiology of invertebrates. Akade 'miai Kiado', Budapest. p. 493-501.

Sokolova, I. M., A., Ringwood, H., Johnson, C. 2005. Tissuespecific accumulation of cadmium in subcellular compartments of eastern oysters Crassostrea virginica. Gmelin (Bivalvia: Ostreidae). Aquat. Toxicol. 74: 218-228.

Theede, H. \& Schoslz, N. 1982. Anreicherung und schadwirkung von cadmium beimeerestieren naturw. rdsch. 35 (7): 286-292.

Vernberg, J. F. 1983. Respiratory adaptations. En: Bliss, D. (editor). The biology of crustacean: Internal anatomy and physiological regulation. New York: Academy press. 8: 1-43.

Winkler, L. 1988. The determination of dissolved oxygen in water. Berlin. Deutschen Chemischen Gesellschaft. 21: 28-43. 